\title{
Identification and characterization of papain-generated antioxidant peptides from palm kernel cake proteins
}

\begin{abstract}
Novel peptides with antioxidant activity were isolated and identified from papain generated palm kernel cake (PKC) proteolysate. The proteolysate was fractionated into individual peptides based on hydrophobicity and isoelectric point using reversed-phase highperformance liquid chromatography and isoelectric focusing techniques. The individual peptides were identified by tandem mass spectrometry and their respective antioxidant activities were evaluated using 2,2-diphenyl-1-picrylhydrazyl radical scavenging activity and metal chelating activity assays. Peptide sequences, AWFS, WAF, and LPWRPATNVF showed the highest radical scavenging activities of $71 \%, 56 \%$, and $50 \%$, respectively, while peptide sequences GGIF, YGIKVGYAIP and YLLLK showed the highest metal chelating activities of 56\%, 53\%, and 50\%, respectively. However, the best IC50 values of peptides measured by DPPHradical dot assay were displayed by GIFE, GVQEGAGHYALL and GGIF at $0.02 \varepsilon M, 0.09 \varepsilon M$ and $0.35 \varepsilon \mathrm{M}$, respectively, while the best half maximal inhibitory concentration values measured using metal chelating activity were shown by LPWRPATNVF, AWFS and YGIKVGYAIP at $0.001 \varepsilon \mathrm{M}, 0.002 \varepsilon \mathrm{M}$ and $0.087 \varepsilon \mathrm{M}$, respectively. It can be concluded that the peptides derived from PKC proteolysate were more potent and distinctive compared to those previously reported from other plant protein sources.
\end{abstract}

Keyword: Antioxidant; Palm kernel cake; Protelysates; Bioactive peptide; Metal chelating activity; Radical scavenging activity 